\title{
Quelles sont les pratiques de prescription des médecins de soins de santé primaires au Canada pour le traitement de la gonorrhée?
}

\author{
$\mathrm{S} \mathrm{Ha}^{1}$, L Pogany ${ }^{2}$, J Seto ${ }^{3}$, J Wu ${ }^{4}, \mathrm{M} \mathrm{Gale-Rowe}^{4 *}$
}

\section{Résumé}

Contexte : Les cas de Neisseria gonorrhea sont à la hausse au Canada, et si elle n'est pas détectée ou sous-traitée, elle peut conduire à une morbidité ou à l'infertilité. En outre, le nombre de souches résistantes aux antimicrobiens est également à la hausse, ce qui crée le risque que N. gonorrhea devienne incurable. En 2013, l'Agence de la santé publique du Canada (ASPC) a publié des recommandations canadiennes pour la prise en charge et le traitement de la gonorrhée qui ont établi la nécessité de la polythérapie pour contrer et réduire la résistance aux antimicrobiens. Toutefois, le degré de sensibilisation et d'adoption de ces recommandations n'est pas bien connu.

Objectifs : Évaluer les pratiques de prescription des médecins de soins de santé primaires pour la prise en charge et le traitement de la gonorrhée.

Méthodologie : Après les essais de validité, deux enquêtes transversales en ligne ont été réalisées au moyen d'un échantillon de commodité des médecins canadiens. Les médecins ont répondu à des énoncés "vrai ou faux » et à des questions ouvertes relativement à trois scénarios cliniques : 1) cas soupçonné d'une infection anogénitale en s'appuyant sur une population d'hommes ayant des relations sexuelles avec d'autres hommes (HARSAH); 2) cas soupçonné d'une infection anogénitale en s'appuyant sur une population autre que d'hommes ayant des relations sexuelles avec d'autres hommes et 3 ) cas soupçonné d'une infection pharyngée en s'appuyant sur toute la population. Les fréquences des réponses ont été calculées pour les énoncés. Les réponses aux questions ouvertes ont été reprogrammées dans des catégories de traitement et les fréquences ont été calculées pour chaque scénario.

Résultats : Au total, 625 médecins ont répondu à l'enquête. La plupart des médecins (de $60 \%$ à $95 \%$ ) ont correctement identifié les énoncés de connaissances concernant la gestion des produits pharmaceutiques, la notification aux partenaires et la déclaration à la santé publique. Pour tous les scénarios cliniques, entre $30 \%$ et $35 \%$ des médecins n'ont fourni aucun renseignement sur le traitement, environ $30 \%$ ont déclaré qu'ils traitaient avec la monothérapie à base de céphalosporines, de $20 \%$ à $25 \%$ ont dit qu'ils prescriraient un traitement à base de céphalosporines et d'azithromycine et une minorité de médecins a nommé d'autres options de traitement. Lorsque les médecins ont été interrogés sur le but du deuxième antibiotique, l'azithromycine, $49 \%$ d'entre eux ont indiqué qu'il servait à offrir un traitement de présomption pour la gonorrhée et la chlamydia. Ils ont indiqué dans une proportion de $41 \%$ qu'il visait à offrir un traitement de présomption pour la chlamydia seulement.

Conclusion : Cet échantillon de commodité porte à croire que malgré un niveau de connaissance de la gestion des produits pharmaceutiques, de la notification aux partenaires et de la déclaration à la santé publique élevé, le recours à la polythérapie pour entraver le développement de la gonorrhée résistante aux antimicrobiens n'est peut-être pas répandu parmi les médecins de premier recours. À la lumière de l'incidence croissante de N. gonorrhea et de l'augmentation des taux de résistance aux antimicrobiens au Canada, une réflexion quant à la façon d'améliorer la sensibilisation et l'adoption de pratiques exemplaires en matière de prescription en soins primaires pourrait être de mise.

\author{
Affiliations \\ ${ }^{1}$ Direction générale des produits \\ de santé et des aliments, \\ Santé Canada, Ottawa (Ontario) \\ ${ }^{2}$ Direction générale de la \\ réglementation des opérations \\ et des régions, Santé Canada, \\ Ottawa (Ontario) \\ ${ }^{3}$ Affaires mondiales Canada, \\ Ottawa (Ontario) \\ ${ }^{4}$ Centre de la lutte contre les \\ maladies transmissibles et les \\ infections, Agence de la santé \\ publique du Canada, Ottawa \\ (Ontario)
}

*Correspondance : margaret. gale-rowe@phac-aspc.gc.ca 


\section{Introduction}

L'infection gonococcique, causée par Neisseria gonorrhea, est un problème clinique et de santé publique croissant en raison de l'augmentation des taux, des profils de résistance aux antimicrobiens et de son association à des séquelles sur la santé à long terme lorsqu'elle n'est pas traitée ou traitée inefficacement. Chez les femmes, une gonorrhée non traitée est associée à l'infection génitale haute, aux grossesses ectopiques ou à l'infertilité; chez les hommes, elle est liée à l'épididymite ou à l'infertilité $(1,2)$. Au Canada, les cas déclarés de gonorrhée ont augmenté de 38,9\% entre 2003 et 2012, les taux les plus élevés étant enregistrés chez les hommes âgés de 20 à 24 ans $(148,5$ par tranche de 100000$)$ et les femmes (153,0 par tranche de 100 000) (3).

Outre l'augmentation des taux de gonorrhée, le nombre de cas de N. gonorrhea a augmenté (4), y compris les souches résistantes à la ceftriaxone (5). Le gouvernement du Canada a déterminé que la résistance aux antimicrobiens était un domaine d'action prioritaire et en 2013, I'Agence de la santé publique du Canada (ASPC) a publié des recommandations mises à jour pour le traitement de la gonorrhée dans ses Lignes directrices canadiennes sur les infections transmissibles sexuellement. Ces lignes directrices recommandent une thérapie à base d'antibiotiques, le choix des médicaments variant selon la population et le site d'infection $(6,7)$ (tableau 1). La polythérapie à base d'antibiotiques est recommandée comme thérapie privilégiée. Elle assure un traitement aux antibiotiques agissant par l'intermédiaire de deux mécanismes différents, ce qui réduit la probabilité d'échec du traitement, contre l'émergence de la gonorrhée résistante à plusieurs médicaments et traite efficacement la chlamydia (8-12). Toutefois, d'autres lignes directrices sont disponibles et peuvent orienter la pratique des médecins de premier recours.

Les constatations des cliniques de santé sexuelle du Canada laissent entendre que la polythérapie est prescrite au moins $76 \%$ du temps (13); on manque toutefois de données probantes documentant les pratiques de prescription des médecins de premier recours. Les professionnels des soins de santé primaires jouent un rôle important dans la prévention et la prise en charge de la gonorrhée résistante aux antimicrobiens.

Les objectifs de l'étude étaient de décrire les connaissances des médecins de soins de santé primaires en ce qui concerne la prise en charge de la gonorrhée résistante aux antimicrobiens et d'établir leur préférence en matière de prescription pour trois scénarios cliniques.

\section{Méthodologie}

\section{Enquête}

L'ASPC a commandé deux enquêtes transversales en ligne auprès d'une entreprise effectuant des enquêtes en ligne et qui recrute des médecins partout au Canada qui acceptent d'être contactés pour des enquêtes. En avril 2014 et en mars 2015, les médecins participants ont reçu une invitation à participer à une enquête en ligne de 20 minutes comportant des questions ouvertes et fermées. Les questions de l'enquête provenaient de questionnaires précédemment conçus, et leur
Tableau 1 : Lignes directrices canadiennes sur les infections transmissibles sexuellement de 2013 pour le traitement de la gonorrhée non compliquée

\begin{tabular}{|c|c|c|}
\hline Scénario & Thérapie privilégiée & Thérapie parallèle \\
\hline $\begin{array}{l}\text { Adultes et jeunes } \\
\text { autres qu'un } \\
\text { HARSAH ( } 9 \text { ans ou } \\
\text { plus) présentant une } \\
\text { infection anogénitale } \\
\text { non compliquée }\end{array}$ & $\begin{array}{l}\text { Ceftriaxone à raison } \\
\text { de } 250 \text { mg i.m. PLUS } \\
\text { azithromycine à raison de } \\
1 \mathrm{~g} \text { par voie orale } \\
\text { OU } \\
\text { Céfixime à raison } \\
\text { de } 800 \text { mg PLUS } \\
\text { azithromycine à raison de } \\
1 \mathrm{~g} \text { par voie orale }\end{array}$ & $\begin{array}{l}\text { Spectinomycine à } \\
\text { raison de } 2 \mathrm{~g} \text { i.m. PLUS } \\
\text { azithromycine à raison } \\
\text { de } 1 \mathrm{~g} \\
\mathrm{OU} \\
\text { Azithromycine à raison de } \\
2 \mathrm{~g} \text { par voir orale }\end{array}$ \\
\hline $\begin{array}{l}\text { Adultes et jeunes } \\
\text { autres qu'un } \\
\text { HARSAH ( } 9 \text { ans ou } \\
\text { plus) présentant une } \\
\text { infection pharyngée } \\
\text { non compliquée }\end{array}$ & $\begin{array}{l}\text { Ceftriaxone à raison } \\
\text { de } 250 \text { mg i.m. PLUS } \\
\text { azithromycine à raison de } \\
1 \mathrm{~g} \text { par voie orale }\end{array}$ & $\begin{array}{l}\text { Céfixime à raison de } \\
800 \mathrm{mg} \text { par voie orale } \\
\text { PLUS azithromycine à } \\
\text { raison de } 1 \mathrm{~g} \text { par voie } \\
\text { orale } \\
\text { OU } \\
\text { Azithromycine à raison de } \\
2 \mathrm{~g} \text { par voir orale }\end{array}$ \\
\hline $\begin{array}{l}\text { Hommes ayant des } \\
\text { relations sexuelles } \\
\text { avec d'autres } \\
\text { hommes (HARSAH) } \\
\text { atteints d'une } \\
\text { infection anogénitale }\end{array}$ & $\begin{array}{l}\text { Ceftriaxone à raison } \\
\text { de } 250 \text { mg par voie } \\
\text { intramusculaire (i.m.) PLUS } \\
\text { azithromycine à raison de } \\
1 \mathrm{~g} \text { par voie orale }\end{array}$ & $\begin{array}{l}\text { Céfixime à raison de } \\
800 \mathrm{mg} \text { par voie orale } \\
\text { PLUS azithromycine à } \\
\text { raison de } 1 \mathrm{~g} \text { par voie } \\
\text { orale } \\
\text { OU } \\
\text { Spectinomycine à } \\
\text { raison de } 2 \mathrm{~g} \text { i.m. PLUS } \\
\text { azithromycine à raison de } \\
1 \mathrm{~g} \text { par voie orale } \\
\text { OU } \\
\text { Azithromycine à raison de } \\
2 \mathrm{~g} \text { par voir orale }\end{array}$ \\
\hline $\begin{array}{l}\text { HARSAH atteints } \\
\text { d'une infection } \\
\text { pharyngée non } \\
\text { compliquée }\end{array}$ & $\begin{array}{l}\text { Ceftriaxone à raison } \\
\text { de } 250 \mathrm{mg} \text { i.m. PLUS } \\
\text { azithromycine à raison de } \\
1 \mathrm{~g} \text { par voie orale }\end{array}$ & $\begin{array}{l}\text { Céfixime à raison de } \\
800 \text { mg par voie orale } \\
\text { PLUS azithromycine à } \\
\text { raison de } 1 \mathrm{~g} \text { par voie } \\
\text { orale }\end{array}$ \\
\hline
\end{tabular}

Abréviations : HARSAH, hommes ayant des relations sexuelles avec d'autres hommes; i.m., par voie intramusculaire; per os, prise par la bouche (par voie orale)

validité apparente a été mise à l'épreuve auprès d'infirmiers, de médecins et d'épidémiologistes de l'ASPC. On a demandé aux médecins de répondre à 14 énoncés " vrai ou faux " concernant l'épidémiologie, le diagnostic, la prise en charge et la déclaration à la santé publique de la gonorrhée résistante aux antimicrobiens. On a sollicité des réponses ouvertes pour trois scénarios cliniques: 1) cas soupçonné d'une infection anogénitale en s'appuyant sur une population d'hommes ayant des relations sexuelles avec d'autres hommes (HARSAH); 2) cas soupçonné d'une infection anogénitale en s'appuyant sur une population autre que d'hommes ayant des relations sexuelles avec d'autres hommes et 3) cas soupçonné d'une infection pharyngée en s'appuyant sur toute la population. Les médecins ont reçu un incitatif financier pour leur participation à l'enquête.

En 2015, on a ajouté une question dans le but de clarifier les raisons des médecins de prescrire le deuxième antibiotique, l'azithromycine, pour le traitement de la gonorrhée.

\section{Analyse des données}

Les données ont été analysées au moyen du logiciel Enterprise Guide de SAS (v5.1). Le test de chi carré de Pearson a servi à comparer les deux échantillons. Les ensembles de 
données provenant des deux cycles d'enquête ont été combinés pour l'analyse puisqu'on n'a constaté aucune différence entre les populations échantillons. La fréquence des bonnes réponses a été calculée pour chacun des énoncés « vrai ou faux " sur le diagnostic, le traitement, le suivi et la déclaration à la santé publique de la gonorrhée. Les réponses ouvertes aux trois scénarios cliniques ont été reprogrammées dans les catégories de traitement suivantes : ceftriaxone et azithromycine; céfixime et azithhromycine; céphalosporine seule; azithromycine seule; spectinomycine combinée à l'azithromycine et autres schémas pharmaceutiques. On a exclu les renseignements posologiques et la voie d'administration en raison des grandes quantités de données manquantes. On a regroupé les médecins qui ont répondu "sans objet ", " je ne traite pas d'hommes ", « je ne suis pas certain » ou « je ne sais pas » sous la catégorie " aucune information sur les traitements".

\section{Résultats}

Un total de 2500 médecins ont été contactés pour la première enquête et 321 ont complété l'enquête pour un taux de réponse de $13 \%$. Un total de 3600 médecins ont été contactés pour la deuxième enquête et 304 ont complété l'enquête pour un taux de réponse de huit pour cent. Au total, 625 médecins ont complété les deux enquêtes.

\section{Données démographiques et caractéristiques des médecins}

Les deux tiers des répondants étaient de sexe masculin (66\%), $83 \%$ des médecins comptaient 10 ans ou plus de pratique et $85 \%$ travaillaient en médecine familiale. Près de $75 \%$ des répondants ont diagnostiqué au moins un cas de gonorrhée au cours de l'année précédente (tableau 2).

Tableau 2 : Caractéristiques socio-démographiques des répondants $(\mathrm{N}=625)$

\begin{tabular}{|c|c|c|}
\hline Caractéristique & $\mathbf{N}$ & (\%) \\
\hline \multicolumn{3}{|l|}{ Sexe } \\
\hline Femme & 208 & $33 \%$ \\
\hline Homme & 410 & $66 \%$ \\
\hline $\begin{array}{l}\text { Je préfère ne pas divulguer } \\
\text { cette information }\end{array}$ & 7 & $1 \%$ \\
\hline \multicolumn{3}{|l|}{ Années de pratique (années) } \\
\hline Moins de 10 & 109 & $17 \%$ \\
\hline 10 ou plus & 516 & $83 \%$ \\
\hline \multicolumn{3}{|l|}{ Profession } \\
\hline Médecine familiale & 532 & $85 \%$ \\
\hline Obstétrique-gynécologie & 60 & $10 \%$ \\
\hline Médecine d'urgence & 21 & $3 \%$ \\
\hline Autre & 12 & $2 \%$ \\
\hline \multicolumn{3}{|l|}{ Milieux de pratique $^{1}$} \\
\hline $\begin{array}{l}\text { Médecine générale ou } \\
\text { familiale }\end{array}$ & 502 & $80 \%$ \\
\hline $\begin{array}{l}\text { Centre de santé } \\
\text { communautaire }\end{array}$ & 73 & $12 \%$ \\
\hline
\end{tabular}

Tableau 2 : Caractéristiques socio-démographiques des répondants $(\mathrm{N}=625)$

\begin{tabular}{|l|r|r|}
\hline \multicolumn{1}{|c|}{ Caractéristique } & N & \multicolumn{2}{|c|}{ (\%) } \\
\hline Milieux de pratique ${ }^{1}$ (suite) & 206 & $33 \%$ \\
\hline $\begin{array}{l}\text { Clinique sans rendez-vous/ } \\
\text { soins d'urgence }\end{array}$ & 67 & $11 \%$ \\
\hline Clinique de santé sexuelle & 31 & $5 \%$ \\
\hline $\begin{array}{l}\text { Services de santé pour } \\
\text { étudiants }\end{array}$ & 26 & $4 \%$ \\
\hline Salle d'urgence & 14 & $2 \%$ \\
\hline $\begin{array}{l}\text { Clinique d'obstétrique et de } \\
\text { gynécologie }\end{array}$ & 36 & $6 \%$ \\
\hline Autre & & \\
\hline
\end{tabular}

Nombre de cas de gonorrhée diagnostiqués au cours de l'année précédente

\begin{tabular}{|l|r|r|}
\hline 0 & 153 & $25 \%$ \\
\hline 1 & 90 & $14 \%$ \\
\hline $2-4$ & 169 & $27 \%$ \\
\hline $5-9$ & 97 & $16 \%$ \\
\hline $10+$ & 104 & $17 \%$ \\
\hline Incertain/aucune réponse & 12 & $2 \%$ \\
\hline
\end{tabular}

Abréviations : N, nombre; \%, pourcentage

Les pourcentages ne totalisent pas $100 \%$ comme les médecins peuvent travailler dans plus d'un milieu. Le dénominateur est la taille totale de l'échantillon

\section{Connaissances liées à la prise en charge de la gonorrhée résistante aux antimicrobiens}

En tout, de $60 \%$ à $95 \%$ des médecins ont correctement identifié les énoncés de connaissances concernant la gestion des produits pharmaceutiques, la notification aux partenaires et la déclaration à la santé publique. Environ les deux tiers des répondants ont correctement identifié les énoncés liés aux tendances actuelles concernant l'incidence croissante, les groupes d'âge les plus fréquemment touchés et la présence de gonorrhée résistante aux antimicrobiens au Canada (tableau 3). La plupart des répondants (87\%) ont indiqué l'importance d'un traitement concomitant pour la chlamydia.

\section{Tableau 3 : Énoncés de connaissances pour la gestion} par la santé publique de la gonorrhée résistante aux antimicrobiens

\begin{tabular}{|l|l|c|}
\hline \multicolumn{1}{c|}{$\begin{array}{c}\text { Région } \\
\text { évaluée }\end{array}$} & \multicolumn{1}{|c|}{ Énoncé de connaissances } & $\begin{array}{c}\text { N (en \%) } \\
\text { de bonnes } \\
\text { réponses }\end{array}$ \\
\hline Épidémiologie & $\begin{array}{l}\text { Le nombre de cas d'infection } \\
\text { gonococcique déclarés a diminué } \\
\text { au cours de la dernière décennie. } \\
\text { (Vrai) }\end{array}$ & 384 (61\%) \\
\cline { 2 - 3 } & $\begin{array}{l}\text { La gonorrhée résistante aux } \\
\text { antibiotiques n'est pas un } \\
\text { problème au Canada. (Faux) }\end{array}$ & $400(64 \%)$ \\
\cline { 2 - 3 } & $\begin{array}{l}\text { La gonorrhée touche surtout les } \\
\text { femmes âgées de 30 à 40 ans. } \\
\text { (Faux) }\end{array}$ & $316(51 \%)$ \\
\hline
\end{tabular}


Tableau 3 : Énoncés de connaissances pour la gestion par la santé publique de la gonorrhée résistante aux antimicrobiens (suite)

\begin{tabular}{|c|c|c|}
\hline $\begin{array}{l}\text { Région } \\
\text { évaluée }\end{array}$ & Énoncé de connaissances & $\begin{array}{l}\mathrm{N} \text { (en \%) } \\
\text { de bonnes } \\
\text { réponses }\end{array}$ \\
\hline \multirow[t]{4}{*}{$\begin{array}{l}\text { Épreuves } \\
\text { diagnostiques }\end{array}$} & $\begin{array}{l}\text { Un test de suivi du traitement } \\
\text { devrait être effectué pour tous les } \\
\text { cas de gonorrhée diagnostiquée, } \\
\text { dans la mesure du possible. (Vrai) }\end{array}$ & 445 (71\%) \\
\hline & $\begin{array}{l}\text { Les cultures sont particulièrement } \\
\text { importantes pour les hommes ayant } \\
\text { des relations sexuelles avec d'autres } \\
\text { hommes asymptomatiques. (Vrai) }\end{array}$ & $413(66 \%)$ \\
\hline & $\begin{array}{l}\text { Quand une infection gonococcique } \\
\text { est soupçonnée, des échantillons } \\
\text { doivent être pris chez des patients } \\
\text { symptomatiques, puis transmis } \\
\text { en vue d'effectuer une culture et } \\
\text { un test d'amplification des acides } \\
\text { nucléiques (TAAN). (Vrai) }\end{array}$ & $350(56 \%)$ \\
\hline & $\begin{array}{l}\text { Les patients pour qui le traitement } \\
\text { échoue devraient repasser un } \\
\text { test d'amplification des acides } \\
\text { nucléiques (TAAN). (Faux) }\end{array}$ & $82(13 \%)$ \\
\hline \multirow[t]{3}{*}{$\begin{array}{l}\text { Gestion des } \\
\text { produits } \\
\text { pharmaceutiques }\end{array}$} & $\begin{array}{l}\text { Un traitement concomitant pour la } \\
\text { chlamydia est conseillé lorsqu'un } \\
\text { patient est traité pour une } \\
\text { gonorrhée. (Vrai) }\end{array}$ & $542(87 \%)$ \\
\hline & $\begin{array}{l}\text { Il est nécessaire d'attendre les } \\
\text { résultats de la culture avant } \\
\text { d'entreprendre un traitement aux } \\
\text { antibiotiques des cas de gonorrhée. } \\
\text { (Faux) }\end{array}$ & 492 (79 \%) \\
\hline & $\begin{array}{l}\text { Les patients atteints de gonorrhée } \\
\text { devraient être traités au moyen } \\
\text { d'une polythérapie. (Vrai) }\end{array}$ & $374(60 \%)$ \\
\hline \multirow[t]{2}{*}{$\begin{array}{l}\text { Notification aux } \\
\text { partenaires }\end{array}$} & $\begin{array}{l}\text { Il est important de signaler } \\
\text { l'infection aux partenaires des } \\
60 \text { derniers jours dans tous les cas } \\
\text { de gonorrhée diagnostiquée. (Vrai) }\end{array}$ & 542 (87 \%) \\
\hline & $\begin{array}{l}\text { Les bureaux de santé publique } \\
\text { peuvent aider les médecins à } \\
\text { annoncer l'infection aux partenaires. } \\
\text { (Vrai) }\end{array}$ & 593 (95\%) \\
\hline \multirow[t]{2}{*}{$\begin{array}{l}\text { Production de } \\
\text { rapports liés à la } \\
\text { santé publique }\end{array}$} & $\begin{array}{l}\text { L'infection gonococcique est une } \\
\text { infection à déclaration obligatoire. } \\
\text { Les autorités locales en matière } \\
\text { de santé publique doivent être } \\
\text { informées rapidement. (Vrai) }\end{array}$ & 595 (95 \%) \\
\hline & $\begin{array}{l}\text { La gonorrhée ne fait pas partie des } \\
\text { infections à déclaration obligatoire } \\
\text { au Canada. (Faux) }\end{array}$ & $463(74 \%)$ \\
\hline
\end{tabular}

Abréviations : HARSAH, hommes ayant des relations sexuelles avec les hommes; TAAN, test d'amplification des acides nucléiques

On ajouté à l'enquête de 2015 une question additionnelle sur la raison de prescrire un deuxième antibiotique, et $49 \%$ des répondants ont indiqué qu'il servait de traitement de présomption pour la gonorrhée et la chlamydia et $41 \%$ ont indiqué qu'il servait aux fins de traitement de présomption pour la chlamydia (figure 1).
Figure 1 : Raisons motivant la prescription par les médecins de famille du deuxième antibiotique pour le traitement de la gonorrhée (2015)

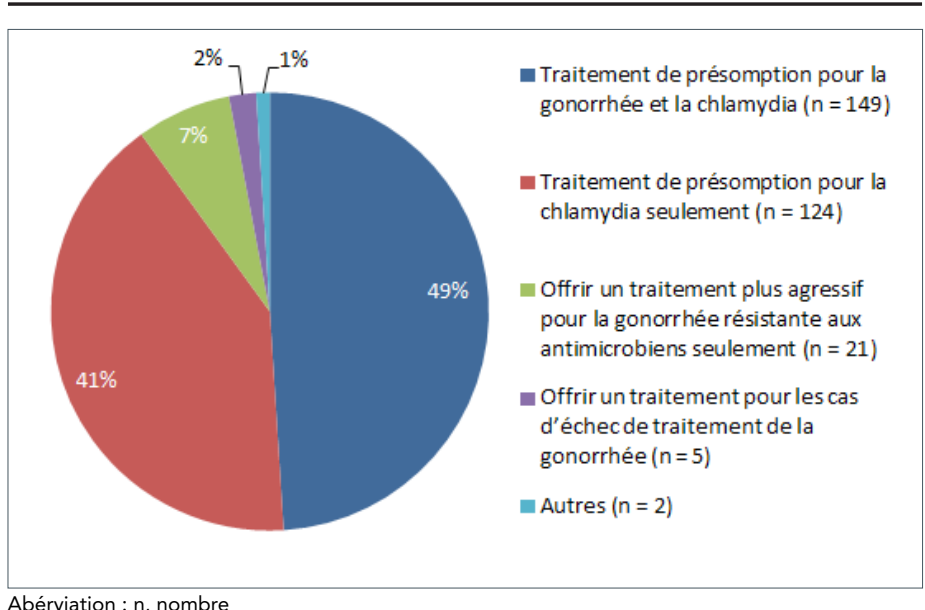

Abérviation : $n$, nombre

\section{Pratiques de prescription des médecins}

Le tableau 4 résume les données liées aux pratiques de prescription. Pour tous les scénarios cliniques, de $30 \%$ à $35 \%$ des médecins n'ont fourni aucune information sur le traitement, environ $30 \%$ des médecins ont déclaré un traitement au moyen de la monothérapie à base de céphalosporines, de $20 \%$ à $25 \%$ ont dit qu'ils prescriraient de la céphalosporine et de l'azithromycine et une minorité de médecins a nommé d'autres options de traitement.

Tableau 4 : Intention de prescription des médecins pour trois scénarios cliniques

\begin{tabular}{|c|c|c|c|}
\hline $\begin{array}{l}\text { Choix de } \\
\text { traitement } \\
\text { rapportés }\end{array}$ & $\begin{array}{c}\text { n (en \%) } \\
\text { d'infections } \\
\text { anogénitales } \\
\text { chez les } \\
\text { HARSAH }\end{array}$ & $\begin{array}{c}n \text { (en \%) } \\
\text { d'infections } \\
\text { anogénitales } \\
\text { chez les } \\
\text { patients autres } \\
\text { qu'un HARSAH }\end{array}$ & $\begin{array}{c}\text { n (en \%) } \\
\text { d'infections } \\
\text { pharyngées }\end{array}$ \\
\hline $\begin{array}{l}\text { Ceftriaxone + } \\
\text { azithromycine }\end{array}$ & $81(13,0 \%)^{1}$ & $74(11,8 \%)^{1}$ & $68(10,9 \%)^{1}$ \\
\hline $\begin{array}{l}\text { Céfixime + } \\
\text { azithromycine }\end{array}$ & $72(11,5 \%)^{2}$ & $84(13,4 \%)^{1}$ & $53(8,5 \%)^{2}$ \\
\hline Azithromycine & $34(5,4 \%)^{2}$ & $50(8,0 \%)^{2}$ & $53(8,5 \%)^{2}$ \\
\hline $\begin{array}{l}\text { Spectinomycine } \\
+ \text { azithromycine }\end{array}$ & $0(0,0 \%)^{2}$ & $0(0,0 \%)^{2}$ & $0(0,0 \%)$ \\
\hline $\begin{array}{l}\text { Céphalosporine } \\
\text { seulement }^{1}\end{array}$ & $179(28,6 \%)$ & $186(29,8 \%)$ & $167(26,7 \%)$ \\
\hline Autres ${ }^{2}$ & $82(13,1 \%)$ & $84(13,4 \%)$ & $93(14,9 \%)$ \\
\hline $\begin{array}{l}\text { Aucune } \\
\text { information sur } \\
\text { les traitements }\end{array}$ & $177(28,3 \%)$ & $147(23,5 \%)$ & $191(30,6 \%)$ \\
\hline
\end{tabular}

En ce qui a trait aux hommes ayant des relations sexuelles avec les hommes présentant une infection anogénitale soupçonnée, près de $30 \%$ des médecins n'ont indiqué aucune option de traitement, près de $30 \%$ ont dit qu'ils prescriraient de la 
céphalosporine seule, $25 \%$ ont indiqué qu'ils prescriraient de la céphalosporine et de l'azithromycine, $5 \%$ ont répondu l'azithromycine seulement, et le reste a nommé d'autres options de traitement. En ce qui concerne les patients autres qu'un homme ayant des relations sexuelles avec les hommes présentant une infection gonococcique anogénitale non compliquée, $30 \%$ des médecins ont dit utiliser la monotéhrapie à base de céphalosporines, $25 \%$ ont indiqué une polythérapie au moyen de céphalosporines et d'azithromcycine; $8 \%$ ont indiqué un traitement au moyen d'azithromycine et $13 \%$ ont dit utiliser d'autres schémas d'antibiothérapies. Pour le traitement des patients atteints d'une infection pharyngée non compliquée, un peu plus de $30 \%$ des médecins n'ont indiqué aucun traitement, $27 \%$ ont indiqué une monothérapie à base de céphalosporines, près de $20 \%$ ont indiqué une polythérapie au moyen de la céphalosporine et d'azithromycine et $15 \%$ des médecins ont dit utiliser d'autres stratégies de gestion des produits pharmaceutiques.

\section{Discussion}

Ces enquêtes en ligne démontrent que les médecins participants étaient bien renseignés sur la gestion des produits pharmaceutiques, la notification aux partenaires et la déclaration à la santé publique de $N$. gonorrhea, mais semblaient moins bien renseignées quant au recours à la polythérapie pour entraver le développement de la gonorrhée résistante aux antimicrobiens. Environ $25 \%$ ont rapporté leur intention de prescrire la polythérapie spécifique pour l'infection anogénitale identifiée dans les Lignes directrices canadiennes sur les infections transmissibles sexuellement comme thérapie privilégiée pour prévenir l'échec du traitement et atténuer le développement de la résistance aux antimicrobiens. Les médecins répondants semblaient moins confiants pour les prescriptions dans les cas de gonorrhée pharyngée. Ceci contraste avec les études des cliniques de santé sexuelle, où il est courant de prescrire une polythérapie (14-17).

Une monothérapie pour le traitement de la gonorrhée n'est pas recommandée comme des cas d'échec du traitement par monothérapie à la céfixime par voie orale ont été recensés au Canada $(12,18,19)$. Dans notre échantillon, environ $30 \%$ des médecins ont indiqué qu'ils traiteraient la gonorrhée au moyen d'une monothérapie à base de céphalosporines; toutefois, près de la moitié des médecins ont déclaré que le but du deuxième antibiotique (souvent l'azithromycine) était de traiter la chlamydia seulement (et non la gonorrhée) et $87 \%$ d'entre eux étaient d'avis qu'un traitement concomitant pour la chlamydia était conseillé lorsqu'un patient est traité pour une gonorrhée. À ce titre, on suppose que les médecins de premier recours prescrivent peut-être une polythérapie, mais dans une large mesure pour couvrir une co-infection possible.

Il faut considérer certaines limites. Le taux de réponse a été très faible et l'échantillon de convenance peut ne pas être représentatif des médecins de soins de santé primaires au Canada. En outre, il se peut que certaines des questions aient manqué de clarté et que les réponses des médecins aux énoncés épidémiologiques et diagnostiques aient été incohérentes avec leur épidémiologie et leurs lignes directrices locales, mais pas avec les statistiques nationales ou les Lignes directrices canadiennes sur les infections transmissibles sexuellement.

\section{Conclusion}

À la lumière de l'incidence croissante de la gonorrhée et de la gonorrhée résistante aux antimicrobiens, une sensibilisation accrue et l'adoption de la polythérapie de façon routinière par les médecins de soins de santé primaires pourraient contribuer à réduire au minimum l'échec du traitement et entraver le développement de la résistance à la gonorrhée résistante aux antimicrobiens.

\section{Remerciements}

Nous tenons à remercier le groupe de travail d'experts pour les Lignes directrices canadiennes sur les infections transmissibles sexuellement de leurs contributions.

\section{Conflit d'intérêts}

Aucun.

\section{Financement}

Ce travail a été appuyé par l'Agence de la santé publique du Canada. Les auteurs n'ont aucune source de financement extérieure supplémentaire à déclarer.

\section{Références}

1. Creighton S. Gonorrhea. Am Fam Physician. 2012;85(6):642643.

2. Miller K. Diagnosis and treatment of Nessieria gonorrhoeae infections. Am Fam Physician. 2006;73(10):1779.

3. Totten S, MacLean R, Payne E. L'infection gonococcique au Canada : 2003 - 2012. Relevé des maladies transmissibles au Canada. 2015;41(2). Disponible à l'adresse : http://www.phacaspc.gc.ca/publicat/ccdr-rmtc/15vol41/dr-rm41-02/surv-2-fra. php.

4. Ebrahim M, Gravel D, Thabet C, Abdesselam K, Paramalingam $\mathrm{S}$, Hyson C. Tendance liées à I'utilisation des antimicrobiens et à la résistance aux antimicrobiens au Canada : 2014. Relevé des maladies transmissibles au Canada. 2016;42(11):22731. Disponible à l'adresse : http://www.phac-aspc.gc.ca/ publicat/ccdr-rmtc/16vol42/dr-rm42-11/ar-02-fra.php.

5. Martin I, Sawatzky P, Liu G, Mulvey M. La résistance aux antimicrobiens de Neisseria gonorrhoeae au in Canada: 2009-2013. Relevé des maladies transmissibles au Canada. 2015;41(2). Disponible à l'adresse : http://www.phac-aspc.gc.ca/ publicat/ccdr-rmtc/15vol41/dr-rm41-02/surv-4-fra.php.

6. Agency de la santé publique du Canada. Lignes directrices canadiennes sur les infections transmissibles sexuellement - Infections gonococciques [Internet]. Ottawa: ASPC; 2013 [mise à jour le 26 sept 2014; consulté le 3 mai 2016]. Disponible 
à l'adresse : http://www.phac-aspc.gc.ca/std-mts/sti-its/cgstiIdcits/section-5-6-fra.php.

7. Pogany L, Romanowski B, Robinson J, Gale-Rowe M, LathamCarmanico C, Weir C. Management of gonococcal infection among adults and youth. Can Fam Physician. 2015;61(10):869.

8. Centers for Disease Control and Prevention. Update to CDC's sexually transmitted diseases treatment guidelines, 2010: oral cephalosporins no longer a recommended treatment for gonococcal infections. MMWR. 2015;61(31):590.

9. Furuya R, Koga Y, Irie S, Ikeda F, Kanayama A, Kobayashi I. In vitro activities of antimicrobial combinations against clinical isolates of Neisseria gonorrhoeae. J Infect Chemother. 2013;19(6):1218.

10. Hottes T, Lester R, Hoang L, McKay R, Imperial M, Gilbert M. Cephalosporin and azithromycin susceptibility in Neisseria gonorrhoeae isolates by site of infection, British Columbia, 2006 to 2011. Sexually Transm Dis. 2013;40(1):46.

11. Pereira $R$, Cole $M$, Ison $C$. Combination therapy for gonorrhoea: In vitro synergy testing. J Antimicrob Chemother. 2013;68(3):640.

12. Schumacher C, Ghanem K. Retreatment rates for uncomplicated gonorrhea infection: comparing ceftriaxone and azithromycin versus ceftriaxone and doxycycline. Sexually Transm Dis. 2013;40(7):539.

13. Singh AE Gratrix J, Martin I, Friedman D, Hoang L, Lester $R$, Metz $G$, et al. Gonorrhea treatment failures with oral and injectable expanded spectrum cephalosporin monotherapy vs. dual therapy at 4 Canadian sexually transmitted infection clinics, 2010-2013. Sexually Transm Dis. 2015;42(6).
14. Gratrix J, Bergman J, Anderson N, Read R, Singh A, Smyczek $P$ [Internet]. Adherence to new treatment guidelines for uncomplicated anogenital and pharyngeal Neisseria Gonorrhea cases in adults in Alberta, Canada. Meeting of the Infectious Diseases Society of America. 2014 [consulté le 24 dec 2016]. Disponible à l'adresse : https://www.researchgate. net/publication/267881937_Adherence_to_New_Treatment Guidelines_for_Uncomplicated_Anogenital_and_Pharyngeal_ Neisseria_Gonorrhea_Cases_in_Adults_in_Alberta_Canada.

15. Lechtenberg R, Samuel M, Bernstein K, Lahiff M, Olson N, Bauer $\mathrm{H}$. Variation in adherence to the treatment guidelines for Neisseria gonorrhoeae by clinical practice setting, California, 2009 to 2011. Sexually Transm Dis. 2014;42(6):331.

16. The Eastern European Sexual and Reproductive Health (EE SRH) Network Antimicrobial Resistance Group. Recommended antimicrobial treatment of uncomplicated gonorrhoea in 2009 in 11 East European countries: implementation of a Neisseria gonorrhoeae antimicrobial susceptibility programme in this region is crucial. Sexually Transm Infect. 2010;86:442.

17. Roxanne K, Stenger M, Weinstock H, Berstein K, Reed M, Schumacher C. Gonorrhea treatment practices in the STD Surveillance Network, 2010-2012. Sexually Transm Dis. 2015;42(1):6.

18. Wetten S, Mohammed H, Yung M, Mercer C, Cassell J, Hughes G. Diagnosis and treatment of chlamydia and gonorrhoea in general practice in England 2000-2011: a population-based study using data from the UK clinical practice research datalink. BMJ Open. 2015.

19. Allen V, Mitterni L, Seah C, Anuradha R, Martin I, Lee C. Neisseria gonorrhoeae treatment failure and susceptibility to cefixime in Toronto, Canada. JAMA. 2013;309(2):163.

\section{Lignes directrices canadiennes sur les infections transmissibles sexuellement}

\section{Application mobile maintenant offerte}
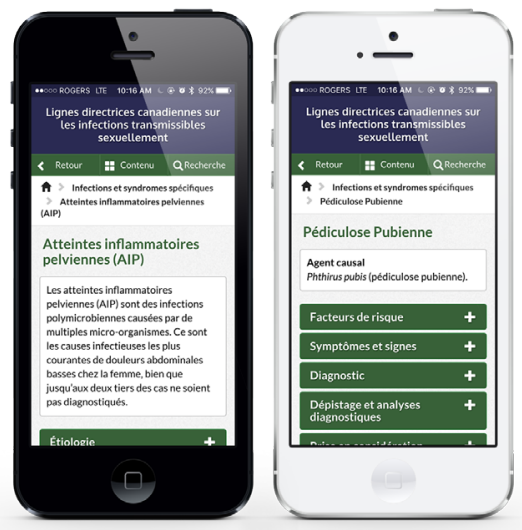

\section{Accès rapide et pratique aux plus récentes lignes directrices canadiennes sur le diagnostic et la prise en charge des ITS.}

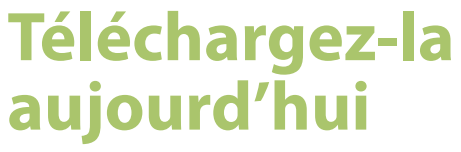

Téléchargement gratuit

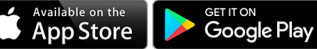

Recherchez « Lignes directrices canadiennes sur les infections transmissibles sexuellement » 